Rwanda Journal of Medicine and Health Sciences Vol.3 No.1, March 2020 https://dx.doi.org/10.4314/rjmhs.v3i1.8

\title{
Assessing Knowledge and Factors Associated to Long Lasting Insecticide Nets use among pregnant women in southern Rwanda
}

\author{
Amos Habimana ${ }^{1 *}$, Malachie Tuyizere ${ }^{1}$, Joseph Gikunju², Dennis Magu ${ }^{2}$ \\ ${ }^{1}$ College of Medicine and Health Sciences, University of Rwanda, P.O. Box 3286 Kigali, \\ ${ }^{2}$ School of Health Sciences Jomo Kenyatta University of Agriculture and Technology, P.O. Box $62000-00200$ \\ Nairobi, Kenya
}

*Corresponding author: Amos Habimana. College of Medicine and Health Sciences, University of Rwanda, P.O. Box 3286 Kigali. Email: habis2012@yahoo.com

\begin{abstract}
\section{Background}

Around 443,000 pregnant women are at risk of malaria each year in Rwanda. LLINs are freely distributed to women at health centers during antenatal care visit and vaccination services.

Methods

A cross-sectional design was used to explore pregnant women's knowledge and factors associated to LLINs use in five cells of Tumba sector. Data was collected through interviews and questionnaires. The data was analyzed using SPSS 21. Bivariate and multivariate analyses were performed with Chi-square test to assess the association between LLINs ownership and utilization of LLINs.

\section{Results}

All respondents had high knowledge and knew that sleeping under LLINs helps to avoid mosquito bites whereas $381(99.2 \%)$ knew that the use of LLIN helps to fight against the burden of malaria. LLIN ownership was 323 $(84.1 \%)$ while usage was $283(87.6 \%)$ among LLINs owners. LLIN ownership is significantly influenced by the level of education $(\mathrm{p}=0.001)$ and utilization $(\mathrm{p}=0.001)$. Although LLINs coverage was high, its utilization was low. Sixty-one respondents (15.9\%) do not have LLINs and $84(22 \%)$ of respondents had low knowledge on LLINs.
\end{abstract}

\section{Conclusion}

Regular training on LLINs may increase awareness of pregnant women on the benefits of LLIN utilization.

Keywords: Malaria, Households, Long-lasting insecticidal nets, Ownership, Rwanda

\section{Background}

Globally, it was estimated that 1.2 billion people were reported to be at high risk of malaria disease in 2014 [1] and more than 11 million of pregnant women living in 38 countries with high transmission in sub-Saharan Africa were infected with malaria. Malaria during pregnancy may impact the health of the fetus leading to preterm birth and low birth weight, neonatal and infant mortality.[2]

In Sub-Saharan Africa where there is intense malaria transmission, malaria accounts for 10,000 deaths in pregnancies and $15 \%$ of all deaths among children less than 59 months every year.[4]

In Rwanda, $90 \%$ of the population is living in highly endemic zones of malaria transmission,[19] comprising a predictable 443,000 pregnant women every year.[4] Between 2010 and 2011, around 6.1 million of LLINs were distributed through the mass campaigns of 15-month for scaling up malaria control, in Rwanda. with this intervention, the country ranked among the top African countries to reach the target of LLINs universal coverage.[7]
Since 2008, there has been a significant reduction of malaria mortality rate from $16.3 \%$ in 2008 to $3.6 \%$ in 2011. [8] Women's knowledge of malaria control remains low in Sub-Sahara African countries. [7] Studies have revealed that heat and not being bothered about mosquito bites, poor memory, lack of bed net, extreme tiredness, sickness, are common factors that affecting and interrupting LLIN utilization. $[9,10]$

The accessibility to LLINs was higher in urban settings $(71 \%)$ than in rural ones $(62 \%)$ across the country and $73 \%$ of pregnant women aged between 15 and 49 years old slept under any bed net.

In pregnancies, LLINs utilization was sight higher in urban $(78 \%)$ than rural regions $(72 \%)$ and among those without formal education, $62 \%$ were not sleeping under a mosquito net compared to those who attended a primary education $(72 \%)$ and/or from colleges $(85 \%)$.

Although it is financial difficult, since 2005, Rwanda has been moving to the use of LLINs rather than ITNs, which is longer lasting than ITNs. [6] As WHO 
recommends LLIN use for achieving universal coverage in malaria control and prevention, the same case Rwanda has adopted the WHO's recommendations for using multiple distribution channels of free LLIN distribution, at antenatal care and immunization services for both pregnant women and children aged 1-59 months respectively. [13]

For reducing malaria spread in Rwanda, the use of LLINs was recommended as the primary strategy of malaria control and prevention. Knowledge about malaria control strategies is a vibrant foregoing aspect for the accepting and utilizing LLINs among community members.[8] Therefore the current study is designed to assess the level of knowledge and factors that can affect LLIN ownership and utilization among pregnant women in Huye district as one of highly malarious regions of Rwanda to support the development of the right interventions for the malaria control and elimination in the vicinity of Huye district.[12]

\section{Methods}

\section{Study design and population}

A cross-sectional quantitative study design that is analytical in nature was used. Among pregnant women in Huye district, southern Rwanda, where $13.4 \%$ of patients attending health facilities had microscopically confirmed malaria.[16]

\section{Sample size determination}

In this study, the calculated sample size (n) was based on Cochran's formula, where the population is infinite: In 1977 Cochran developed a formula to calculate a representative sample for proportions. The sample size was derived from the table for a minimum sample size estimate for a population survey with a $95 \%$ confidence interval.[18] using the following equation:

$Z$ value $= \pm 1.96$ at $95 \%$ confidence interval

$\mathrm{p}=50 \%$ an expected frequency value which is recommended $50 \%$ by Fisher et al (2008) .Since no estimate is available

$\mathrm{q}=1-\mathrm{p}=>1-0.5=0.5$

$\mathrm{d}=5 \%$ : level of precision

Then the following formula was applied:

$\mathrm{n}=Z * Z[\mathrm{p}(1-\mathrm{p}) /(\mathrm{d} * \mathrm{~d})]=1.960 * 1.960[0.50(1-$ $0.50) /(0.05 * 0.05)]=384.16 \approx 384$

Therefore, the sample size was 384 participants, where

The required minimum sample size of 384 pregnant women who visit Rango Health Center of Tumba sector in Huye district for antenatal care and routine immunization has been proportionally computed by villages of Tumba sector

\section{Sampling techniques}

By adopting the balloting approach, the names of the sectors of Huye district were written on small papers, placed and shuffled in a container, with a random selection, one sector (Tumba) was selected from the frame list of 14 sectors in Huye district. Tumba sector was selected at random from the container by a simple random sampling technique. The second stage a list of names of all five villages and the number of households of each village in the Tumba sector based on the 2012 Rwanda Population and Housing Census was found. In the third stage, the streets were randomly selected in the villages afterward systematic sampling was used to select the houses: by an ordered selection of a particular house from the sampling frame. In the sampled houses a woman that was pregnant, was considered and interviewed after signing the consent form. When a woman was not there, the researcher went immediately to the next household on right side for replacement. To be consistent the number of study participants was also underestimated proportionally to each village based on the number of pregnant women visiting antenatal care, vaccination and family planning services per month at Rango health center.

\section{Tool description}

To collect the data, a written semi-structured questionnaire (English) has been used and translated in Kinyarwanda. Slight modifications were made to the questionnaire to fit each of the participants. The research assistants were the four registered nurses; they were daily supervised by the principal investigator to ensure data quality. Written consent to participate was obtained at the beginning of each interview.

During the pilot study, the questionnaire tool was tested in the population from the nearest neighbor sectors: Namely Huye and Ngoma sector. To identify wording problems and having the feedback on potential difficulties when they were answering the questions and filling the form, the participants were given time to ask the questions as the actual study participants. They were requested to identify any challenges they face throughout the questionnaire. It took 25 minutes to complete the questionnaire.

\section{Data collection procedures}

The data were primarily collected using a questionnaire adapted from the questionnaire tool available from the RBM-Rwanda Malaria Indicator Survey of 2017 for returning to the Rwandan population and health issues. Some changes have been decided in collaboration with the team of public health specialists from Rwanda Biomedical 
Centre (RBC). The data were collected by four trained nurses over a period of two months.

\section{Data analysis}

The respondents' LLINs knowledge was graded by one mark (1) and (0) to a correct and wrong answer respectively. Scores range was from 0 to 8 with a mean of $7.1 \quad(\mathrm{SD}=0.83)$. There were three levels of knowledge scores; low scored 0 to 3 , moderate scored 4 to 6 and high knowledge scored 6 to 8 . To find out the relationships between variables, Chisquare statistical tests were computed. SPSS software was used for data analysis.

\section{Ethical considerations}

The study ethical approval was obtained from the Ethics review committee of Jomo Kenyatta University of Agriculture and Technology (JKUAT), Administrative approval has been obtained from Huye district before the study data collection took place. Each and every study participant has signed a written consent form voluntarily. Individual interviews have been done with permission. Every collected data was managed carefully and privately.
To maintain the confidentiality of the collected information, the explanations were given to the study participants. The records from the study participants during an interview have remained confidential only to the researchers.

\section{Results}

\section{Socio-demographic information}

The study participants were 384 pregnant women; the mean age was 30.05 years old, and the class mode of their age was between 25-29 years with 172 $(44.8 \%)$ of respondents. and $215(56 \%)$ of them were married. And also 334 (87\%) of study participants had different levels of education from primary up to high learning institution/college whereas 50 (13\%) never attended the school. It was reported that 294 $(76 \%)$ of the respondents were employed by others, while $76(20 \%)$ of respondents were unemployed and the present study has also shown for most respondents $(228 ; 59.4 \%)$ their income status in terms of money was between 500-1000 Rwf per day. 
Table1. Socio-demographic information of study participants $(n=384)$

\begin{tabular}{|c|c|c|}
\hline Variables & Number $(n=384)$ & Percentage \\
\hline \multicolumn{3}{|l|}{ Age group(Years) } \\
\hline under 24 & 131 & 34.1 \\
\hline $25-29$ & 172 & 44.8 \\
\hline $30-34$ & 67 & 17.4 \\
\hline \multirow{2}{*}{ More than 34} & 14 & 3.6 \\
\hline & Mean age $=30.05$ & $\mathrm{Sd}=7.8$ \\
\hline \multicolumn{3}{|l|}{ Marital status } \\
\hline Single & 215 & 56 \\
\hline Married & 163 & 42.5 \\
\hline Widowed & 2 & 0.5 \\
\hline Divorced & 4 & 1 \\
\hline Total & 384 & 100 \\
\hline \multicolumn{3}{|l|}{ Education level } \\
\hline No formal education & 50 & 13 \\
\hline Primary & 224 & 58.4 \\
\hline Secondary & 100 & 26 \\
\hline HLI/ College & 10 & 2.6 \\
\hline Total & 384 & 100 \\
\hline \multicolumn{3}{|l|}{ Employment status } \\
\hline Women employed by others & 294 & 76 \\
\hline Self-employed women & 14 & 4 \\
\hline Unemployed women & 76 & 20 \\
\hline Total & 384 & 100 \\
\hline \multicolumn{3}{|l|}{ Income status/day } \\
\hline None (ORwf) & 76 & 19.6 \\
\hline 500.5-1000.5 Rwf & 228 & 59.4 \\
\hline 1000.5-1500.5 Rwf & 65 & 17 \\
\hline Above 1500.5 Rwf & 15 & 4 \\
\hline Total & 384 & 100 \\
\hline
\end{tabular}

LLINs Ownership and Utilization among study participants

It is shown in below Table 2 that out of 384 respondents, $323(84.1 \%)$ have been reported as the LLIN owners and most of them $283(87.6 \%)$ were observed as the LLIN utilizers, although 40 (12.4\%) of pregnant women were reported of not utilizing LLIN. Two hundred sixty-four study participants $(81.7 \%)$ were demonstrated to utilizing LLIN among those who owned it. The rest 59 (18.3\%) of them were not sleeping under the LLIN 
Table 2. LLIN Ownership and utilization among respondents

\begin{tabular}{lrr}
\hline LLIN ownership & Number(n=384) & Percentage \\
\hline Yes & 323 & 84.1 \\
No & 61 & 15.9 \\
Total & $\mathbf{3 8 4}$ & $\mathbf{1 0 0}$ \\
LLIN utilization among all respondents & & \\
Yes & 283 & 87.6 \\
No & 40 & 12.4 \\
Total & $\mathbf{3 2 3}$ & $\mathbf{1 0 0}$ \\
Only respondents with LLIN & & \\
Yes & 264 & 81.7 \\
No & 59 & 18.3 \\
Total & $\mathbf{3 2 3}$ & $\mathbf{1 0 0}$ \\
\hline
\end{tabular}

\section{Associations between LLIN ownership and respondent's demographic characteristics}

This study reported that 108 (33.4\%) of respondents aged under 24 owned the LLINs on the first place and followed by those aged between years 30-34 with $59(18.3 \%)$ and lastly those aged more than 34 represented by $14(3.6 \%)$ on the third place of LLIN owners. The association between LLINs ownership and age categories is not statistically significant $(\mathrm{x} 2$ $=3.93 ; p=0.863$ ). It was reported that those who had primary level are the majority of LLIN owners with $222(68.7 \%)$ compared to those who did secondary education with $65(20.1 \%)$, no formal education 29 $(8.9 \%)$ and the least was HLI/college education 7 $(2.1 \%)$ of LLIN ownership. There was an association between level of education and LLINs ownership which is statistically significant $(\mathrm{x} 2=99.8 ; \mathrm{p}=$ 0.001). The association between LLIN ownership and marital status is not statistically significant $(\mathrm{x} 2$ $=1.51 ; \mathrm{p}=0.958)$.
Associations between LLINs Utilization and respondent's demographic characteristics

The present study showed that 127 (44.80\%) participants with group age of 25 to 29 years old have been found as the majority of LLIN utilizers, followed by the other group age of under 24 years old, $95(33.5 \%)$ in second place and then the group aged over 34 years old, 14 (3.6\%). Though not statistically significant $(x 2=2.89 ; \mathrm{p}=0.575)$, it was observed that the respondents aged less than 24 years old were the majority of LLIN owners in relation to those aged between 30 and 34 years old. However, respondents aged between 25-29 years old were the most to utilize LLINs compared with those aged more than 34 years. In addition, the present study has reported that respondents 334 (86.9\%) who attended the school including HLI/college education were the most to utilize the LLIN compared to $50(13.1 \%)$ of those who don't have any level of education $(x 2=72.9$; $\mathrm{p}$-value $=0.001)$. 
Table 3.Relationship between LLIN ownership and utilization with some socio-demographic information

\begin{tabular}{|c|c|c|c|c|c|c|}
\hline Characteristics & $\begin{array}{l}\text { LLIN } \\
\text { ownership }\end{array}$ & $\begin{array}{l}\text { Chi- } \\
\text { square }\end{array}$ & $\begin{array}{l}\mathrm{P}- \\
\text { value }\end{array}$ & $\begin{array}{l}\text { LLIN } \\
\text { utilization }\end{array}$ & $\begin{array}{l}\text { Chi- } \\
\text { square }\end{array}$ & $\begin{array}{l}\mathrm{P} \text { - } \\
\text { value }\end{array}$ \\
\hline \multicolumn{7}{|l|}{ Age group(Years) } \\
\hline under 24 & 108(33.4) & & & $95(33.5)$ & & \\
\hline $25-29$ & $14(4.33)$ & 3.93 & 0.863 & $127(44.8)$ & 2.89 & 0.575 \\
\hline $30-34$ & $59(18.3)$ & & & $52(18.4)$ & & \\
\hline $\begin{array}{c}\text { More than } 34 \\
\text { Marital status }\end{array}$ & $12(3.7)$ & & & $11(3.8)$ & & \\
\hline Single women & $181(56.1)$ & & & $165(58.3)$ & & \\
\hline Married women & $137(42.4)$ & 1.51 & 0.958 & $110(38.8)$ & 1.59 & 0.660 \\
\hline widowed women & $2(0.62)$ & & & $2(0.7)$ & & \\
\hline $\begin{array}{l}\text { Divorced women } \\
\text { Education level }\end{array}$ & $3(0.93)$ & & & $6(2.1)$ & & \\
\hline No formal education & 29(8.9) & & & $23(8.1)$ & & \\
\hline \multicolumn{7}{|l|}{ level } \\
\hline Primary level & $222(68.7)$ & 99.84 & 0.000 & 195(69.0) & 72.9 & 0.001 \\
\hline Secondary level & $65(20.1)$ & & & $58(20.5)$ & & \\
\hline HLI/ College level & $7(2.1)$ & & & $7(2.5)$ & & \\
\hline
\end{tabular}

\section{Knowledge on LLINs}

Scores range was from 0 to 8 with a mean of 7.1 $(\mathrm{SD}=0.83)$. There were three levels of knowledge scores; low scored 0 to 3 , moderate scored 4 to 6 and high knowledge scored 6 to 8 . The present study has shown that all participants have demonstrated that the use of LLIN/sleeping under bed net prevent/avoid the mosquito bites. Among 384 study participants, $381(99.2 \%)$ of them knew that they can use LLINs just to avoid the burden of malaria and $378(98.4 \%)$ agreed also that sleeping under LLIN can prevent malaria transmission to the pregnant women. Additionally, 122 (31.8\%) of study participants demonstrated that LLINs were factorymade with insecticides with the expiration period of four to five years and $110(28.6 \%)$ believed that LLINs are often being washed. 


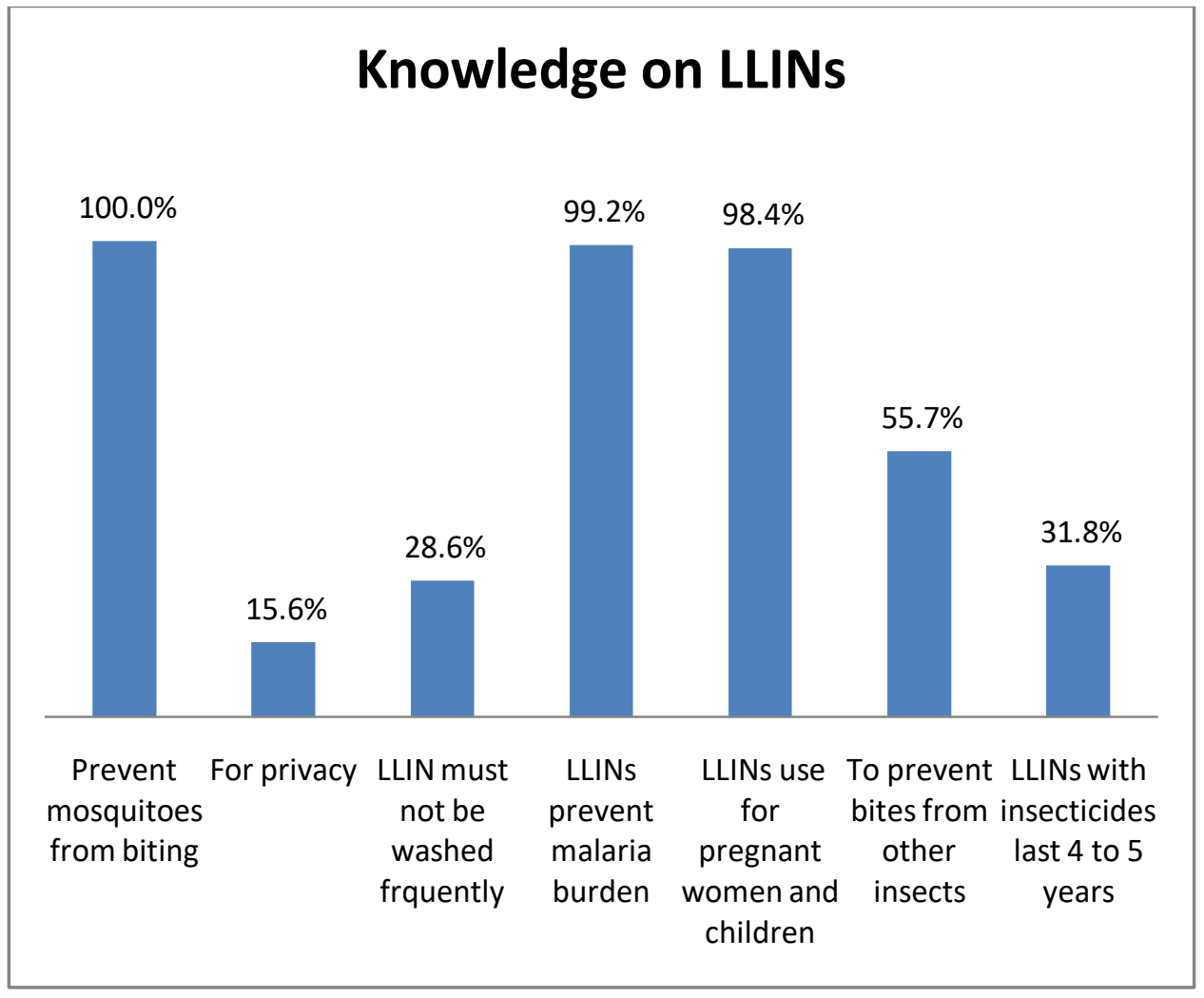

Figure 1. LLIN Knowledge

\section{Factors for not using LLINs}

Among 384 study participants, 59 (18.3\%) were reported not using LLIN. Observed frequent factors that affect LLIN use were included: high temperature/heat 56 (94.9\%), no access to LLIN 52 $(88.1 \%)$ and used mosquito coil/spray 8 (13.6\%). Other factors were included: the absence of mosquitoes 15 (25.4\%), feel uncomfortable sleeping under LLIN $20(33.9 \%)$ and the rest use mosquito coil/spray $8(13.6 \%)$ causes the skin rashes/ itch $42(71.2 \%)$ reduces ventilation /asphyxia 45 $(76.3 \%)$ 


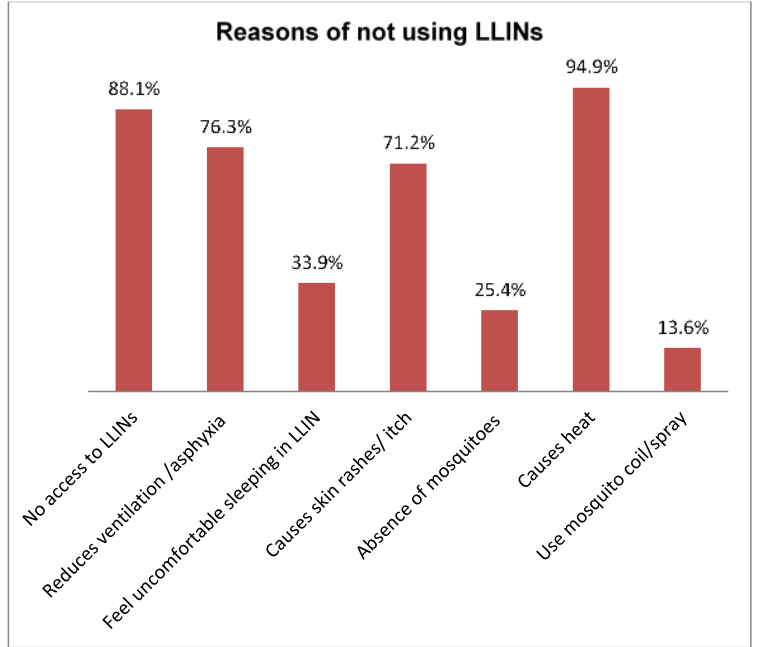

Figure 2. Reasons of not using LLINs

\section{Discussion}

To reach LLINs universal coverage, Rwanda has led down one specific objective of scaling up the distribution of LLINs by targeting the entire population where all households own at least one LLIN. The 2014-2015 DHS has reported $83 \%$ of having at least one LLIN per household while the RMIS 2013 showed ownership of $84 \%$. It was reported that $68 \%$ of children aged between 0-59 months and $70 \%$ of pregnant women sleep under a mosquito net. Moreover, it mentioned that only 61.5 $\%$ of households were utilizing LLINs, this indicates that Rwanda is still far from its objective. Therefore the present study was conducted to figure out LLINs ownership level and its utilization level among pregnant women in Tumba sector, Huye district southern province as one of high malaria transmission region in Rwanda.

\section{LLIN Owners}

The current study has reported 323 (84.1\%), LLIN owners, among the study participants although its findings are not reflecting WHO target of maintaining universal coverage for all age groups at $100 \%$, but findings from this study have shown an increased number of LLINs owners. With regards to RDHS 2014-15 Rwanda Demographic Health Survey, the total ownership of LLINs was $70 \%$ among pregnant women, while the present study has reported a high proportion of LLIN ownership $84.1 \%$ among pregnant women.

\section{LLIN Utilizers}

In the present study, only persons observed to sleep under net were considered as LLINs utilizers. Among 323 study participants of LLIN owners, only $59(18.3 \%)$ were reported to sleep under LLINs. With regards to the present study's' results, the goal set by the Rwanda National malaria control program NMCP of $85 \%$ LLINs utilization was not yet met.[19]. RDHS 2014-15 reports differences in LLINs utilization among those from rural regions and those from urban regions, out of $70 \%$ of pregnant women who utilized LLINs, $41 \%$ were from rural regions while $18 \%$ were from urban regions. [6] Out of $73 \%$ pregnant women who utilized LLINs, with $72 \%$ of them were from rural and $78 \%$ from urban regions, differently the current study has reported $283(87.6 \%)$ of LLIN utilizers, which is quite superior to $73 \%$, which was presented in RDHS 2014-2015. It was also reported that in the southern province including Huye district, $66 \%$ of under-five children slept under ITN whereas paucity findings in terms of LLINs utilization were noticed among the pregnant women living in Huye district. Besides no information among pregnant women who slept under LLIN within rural and urban regions of Huye district, southern province.[20] According to the reports from DHS 2005 and DHS 2010 bed net use in pregnancies rose up from $17 \%$ to $72 \%$ respectively.[19] Briefly, LLINs utilization was reported to be higher in urban regions than rural ones in Rwanda.

\section{Knowledge on LLIN}

The present study has reported that 219 (57\%) of respondents as highly knowledgeable on LLINs whereas $88(23 \%)$ had fair knowledge. Likewise, $(99.2 \%)$ study participants recognized that LLINs were used to avoid the bites of mosquitoes. Different training and health education given by health workers and community health workers at health centers and within the community as supported in their study were the result of respondents' high level of knowledge.[21] The pregnant women were reported to be not knowing that insecticides are infused in LLINs, and that it is contraindicated to wash them often as this was also supported by some researchers.[10] LLINs are being frequently washed due to the low level of knowledge of concerned people which can affect its efficacy. It was shown that health education at health centers does not insist nor stress on the identified gap towards LLIN of not being washed frequently because it is impregnated with insecticides. Hitherto, it considered as a factor of poor or not using LLINs.

\section{Factors influencing LLINs use}

Different studies conducted by [22] have proven that education status, age group, employment status, and income status as some factors that affect LLINs utilization in pregnancies. A study conducted in Cameroon, has shown that $45 \%$ of multiparous women were slept under LLINs, while 21 per cent were primigravida women.[23] Contrary to other studies that showed no significant associations 
between the identified factors and LLINs utilization, it seems that the inconsistencies are due to environmental factors.[24] In Rwanda, wealth status was reported as an influencing factor of LLIN utilization among under-five children.[25] However, paucity studies were done about LLINs utilization among pregnant women.

Though, the current study showed that the respondents aged 25-29 years old were the majority to utilize LLINs in relation to those age of more than 34 years old $(x 2=2.89 ; p=0.575)$ (Table 4$)$. With regards to participants' education level, $7(2.5 \%)$ study participants with HLI/college education were reported to highly use LLIN paralleled with $23(8.1 \%)$ of those who did attend the school $(\mathrm{x} 2=72.9$; $\mathrm{p}=$ $0.001)$.

\section{Barriers to LLIN utilization}

It was reported in the present study that, heat 56 (94.9\%), lack of LLINs 52 (88.1\%), use of mosquito coil and spray $8(13.6 \%)$, no mosquitoes in place of residence 15 (25.4\%), and Feel uncomfortable sleeping under LLIN 20(33.9\%), and the rest utilizing sprays of mosquitoes $8(13.6 \%)$ Causes skin rashes/itch $42 \quad(71.2 \%)$ reduces ventilation/asphyxia $45(76.3 \%)$ as the common barriers that can cause the irregularities in LLIN utilization among those who owned LLINs. Main reasons that were given by study participants for not utilizing LLINs were like heat, no LLIN/unwashed LLIN or torn. It has been also supported by one study conducted by [12] which reported forgetfulness, absence of bed nets, unwashed LLINs or not, tiredness, and sickness as reasons for not utilizing LLINs.[26] Another study has also found heat as a major factor in using LLIN among pregnant women. [10]

\section{Limitations}

The researcher has found this study to be limited in scope and methodology. Study external validity was affected by small sample size. The used crosssectional study design cannot generalize the findings of the study participants' knowledge of LLINs utilization to the entire population. Used structured questionnaire tool can affect and can limit people's responses while collecting data, in this case, study participants may feel limited and challenged when they are making choice throughout the questionnaire and lose the chance for in-depth respond. As malaria is largely an important topic, there were some areas that were left not explored. Some studies done elsewhere were quoted in this study and also reports were undertaken many years ago, henceforward some observed health interventions related to malaria control strategies among pregnant women are nowadays new and changed. To have LLINs or not, to see if they were hung them, were not effectively explored owing to the reason that some data collectors were not given permission to enter and observe LLINs indoor, therefore the findings were mainly based on LLIN owners and utilizers' reports even if 94\% of respondents have been reached at their homes.

\section{Conclusion}

Findings presented in this study are essentials and need to be shared carefully. Nevertheless, the study highlights the need for better using LLIN regularly. Pregnant women's training on LLIN ownership and utilization was observed as crucial to addressing the gap in knowledge revealed in the study. The present study showed that respondents are aware of their responsibilities; nevertheless, low level of knowledge was found along with LLINs utilization and its manipulation among study participants who were not aware that insecticides are infused in LLINs and it is contraindicated to wash them frequently, so far training on LLINs are required to bridge that gap

\section{Recommendations}

Through the refreshment courses and training of health workers and community health workers, Rwanda Ministry of Health needs to make sure that these groups at high risk are aware of what is in the bed net and how it works. In general, there is a need for more researches to conclude why LLIN utilization is not good enough among respondents. To replace the worn bed nets and distributing free LLINs, the government of Rwanda should keep distributing the free LLINs at health centers and within the communities for malaria control and elimination.

\section{Acknowledgements}

The authors express their appreciation to the Head of Rango Health Centre for his cooperation. They are thankful to Prof. Gikuvi Gideon (COD, Department of environmental health and disease control at JKUAT) for administrative support.

\section{Declaration}

The authors declare no conflict of interest in relation to presentation of this manuscript.

\section{Authors' contributions}

HA: Research idea, implementation and manuscript writing.

TM: Manuscript writing

GJ: Study protocol design, implementation

MD: Research proposal

This article is published open access under the Creative Commons Attribution-

NonCommercialNoDerivatives (CC BYNC-ND4.0). 
People can copy and redistribute the article only for noncommercial purposes and as long as they give appropriate credit to the authors. They cannot distribute any modified material obtained by remixing, transforming or building upon this article. See https://creativecommons.org/licenses/by-ncnd/4.0/

\section{References}

1. Hollyman S. WHO | 10 Facts on Malaria. Fact Sheet. 2015.

2. World Health Organization W. World Malaria Report 2018 Isbn 978924156565 3. WHO. 2018;

3. WHO. - World Malaria Report 2013. WHO. 2013.

4. Eckert E, Florey LS, Tongren JE, Salgado SR, Rukundo A, Habimana JP, et al. Impact evaluation of malaria control interventions on morbidity and all-cause child mortality in Rwanda, 2000-2010. Am J Trop Med Hyg. 2017;

5. Gahutu J-B, Steininger C, Shyirambere C, Zeile I, Cwinya-Ay N, Danquah I, et al. Prevalence and risk factors of malaria among children in southern highland Rwanda. Malar J. BioMed Central Ltd; 2011;10:134.

6. National Institute of Statistics of Rwanda M of HR. Rwanda Demographic and Health Survey, 2014-2015. ICF Int. 2016.

7. Goshu YA, Yitayew AE. Malaria knowledge and its associated factors among pregnant women attending antenatal clinic of Adis Zemen Hospital, North-western Ethiopia, 2018. PLoS One. 2019;

8. Adebayo AM, Akinyemi OO, Cadmus EO. Knowledge of malaria prevention among pregnant women and female caregivers of under-five children in rural southwest Nigeria. PeerJ. 2015;

9. Akaba GO, Otubu JAM, Agida ET, Onafowokan O. Knowledge and utilization of malaria preventive measures among pregnant women at a tertiary hospital in Nigeria's federal capital territory. Niger J Clin Pract. 2013;

10. Axame WK, Kweku M, Amelor S, Kye-Duodu G, Agboli E, Agbemafle I, et al. Ownership and Utilization of Long Lasting Insecticide Treated Nets (LLIN) and Factors Associated to Non-utilization Among Pregnant Women in Ho Municipality of Ghana. Cent African J Public Heal. 2016;
11. Kateera F, Ingabire CM, Hakizimana E, Rulisa A, Karinda P, Grobusch MP, et al. Long-lasting insecticidal net source, ownership and use in the context of universal coverage: A household survey in eastern Rwanda. Malar. J. 2015.

12. Tassew A, Hopkins R, Deressa W. Factors influencing the ownership and utilization of longlasting insecticidal nets for malaria prevention in Ethiopia. Malar J. 2017;

13. WHO Global Malaria Programme. Achieving and maintaining universal coverage with long-lasting insecticidal nets for malaria control. Who. 2017;

14. NISR. Fourth Population and Housing Census, Rwanda, 2012. DHS Progr ICF Int Rockville, Maryland, USA. 2012;

15. [Rwanda] MOFH. REPUBLIC OF RWANDA. Health Care (Don. Mills). 2009.

16. Gahutu J-B, Steininger C, Shyirambere C, Zeile I, Cwinya-Ay N, Danquah I, et al. Prevalence and risk factors of malaria among children in southern highland Rwanda. Malar J. BioMed Central Ltd; 2011;10:134.

17. Omair A. Selecting the appropriate study design for your research: Descriptive study designs. J Heal Spec. 2015;

18. Lwanga S, Lemeshow S. Sample size determination in health studies: A practical manual, 1991. World Heal Organ Geneva. 1991;

19. President's Malaria Initiative. FY 2014 Rwanda Malaria Operational Plan. Pres Malar Initiat. 2014;

20. RDHS 2016. Demographic and Health Survey 2014-15: Western Province. Natl. Inst. Stat. Rwanda. 2016.

21. Habimana A, Harerimana A, Asingizwe D, Nyandwi T, Njunwa KJ. Community Health Workers' knowledge, attitudes and practices about malaria prevention in Gicumbi District, Rwanda. Rwanda J [Internet]. 2016;3. Available from: http://www.ajol.info/index.php/rj/article/view/14 7018

22. Nkoka O, Chuang TW, Chuang KY, Chen YH. Factors associated with insecticide-treated net usage among women of childbearing age in Malawi: A multilevel analysis. Malar J. 2018; 
23. Fokam EB, Ngimuh L, Anchang-Kimbi JK, Wanji S. Assessment of the usage and effectiveness of intermittent preventive treatment and insecticidetreated nets on the indicators of malaria among pregnant women attending antenatal care in the Buea Health District, Cameroon. Malar J. 2016;

24. Hill J, Hoyt J, van Eijk AM, D'Mello-Guyett L, ter Kuile FO, Steketee R, et al. Factors Affecting the Delivery, Access, and Use of Interventions to Prevent Malaria in Pregnancy in Sub-Saharan Africa: A Systematic Review and Meta-Analysis. PLoS Med. 2013;
25. Ruyange MM, Condo J, Karema C, Binagwaho A, Rukundo A, Muyirukazi Y. Factors associated with the non-use of insecticide-treated nets in Rwandan children. Malar J. 2016;

26. Muhumuza E, Namuhani $\mathrm{N}$, Balugaba $\mathrm{BE}$, Namata J, Kiracho EE. Factors associated with use of malaria control interventions by pregnant women in Buwunga subcounty, Bugiri District. Malar J. 2016; 